\title{
Die Politik vor der Wissenschaft retten
}

\author{
Politisch rationale Entscheidungen lassen sich nicht durch ausschließlichen \\ Rekurs auf wissenschaftliches Wissen garantieren. Alexander Bogner
}



PRIVATDOZENT DR. ALEXANDER BOGNER

Soziologe, Senior

Scientist Institut für

Technikfolgen-

Abschätzung, Österreichische Akademie der Wissenschaften

Der Text ist Bogners Buch Die Epistemisierung des Politischen.

Wie die Macht des Wissens die Demokratie gefährdet entnommen, das soeben im Reclam Verlag in einer Hardcover-Ausgabe (ISBN: 978-3-15-011343-1) und in der Universalbibliothek (ISBN: 978-3-15014083-3) erschien.

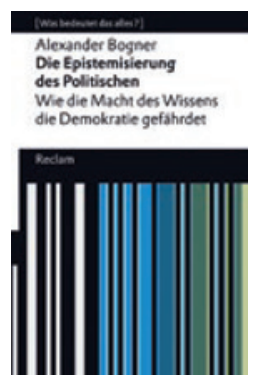

In der Wissensgesellschaft werden viele politische Konflikte als Auseinandersetzungen um das richtige Wissen ausgetragen. Wissenschaftliche Expertise stellt die zentrale Ressource dar, wenn es etwa um die Bewältigung der Klimakrise geht, um den Einsatz von Pestiziden in der Landwirtschaft (Glyphosat), um Grenzwerte der Feinstaubbelastung, um Risiken elektromagnetischer Felder (5G-Netz) oder um die Frage der richtigen Ernährung. Gestritten wird dann in erster Linie um die Zuverlässigkeit von Daten, die Glaubwürdigkeit von Szenarien und Modellen oder die Stichhaltigkeit von Grenzwerten und Kennzahlen - auch wenn eigentlich miteinander rivalisierende Werte und Interessen auf dem Spiel stehen. Wer also an solchen politischen Konflikten ernsthaft teilhaben will, muss über ziemlich profundes Wissen verfügen. Für den jedoch, der es nicht schafft, die eigene normative Position durch den Rekurs auf Expertenwissen abzustützen, wird es eng. Ein Ausweg besteht offensichtlich darin, gegen die etablierte Faktenwelt zu opponieren. Politisch motivierter Protest konzentriert sich damit auf die "Sachfrage“, ob die wissenschaftlichen Fakten denn nicht Fake seien. Dies ließ sich auch im Zuge der Coronakrise beobachten: Auf Grund des hohen Verwissenschaftlichungsdrucks entlud sich mancherorts der Wille zur Fundamentalopposition in der Verbreitung „alternativer Fakten“...

Die größere Sichtbarkeit von Verschwörungstheorien und das Aufblühen der Fake-News-Kultur im Zuge der Coronakrise lassen sich als ideologische Reaktionen auf eine Politik verstehen, die im Einvernehmen mit Epidemiologie und Virologie handelte und sich daher im Rahmen eines Wissenskonflikts nicht wirkungsvoll attackieren ließ. So waren die Proteste nicht nur gegen die Politik und ihre Maßnahmen gerichtet, sondern wiesen immer auch eine antiwissenschaftliche Schlagseite auf: Es ging gegen eine (vermeintlich) autoritative Instanz, die mittels überlegener Rationalität festzulegen beanspruchte, was real, was rational und politisch geboten ist. Alternative Fakten haben ganz offensichtlich Konjunktur, wenn Politik (auf Grund ihrer Übereinstimmung mit der Wissenschaft) als alternativlos erscheint ... Tatsächlich hat die Politik im Kontext erfolgreich durchgesetzter Wissensansprüche der Wissenschaft zumeist nur mehr einen geringen Handlungsspielraum ...
Der Bewegung der Leugner geht es um die Verhinderung einer progressiven Politik, im Fall von Corona nicht anders als im Klimabereich. Aus Angst davor, dass weitreichender Expertenkonsens die Politik $\mathrm{zu}$ Regulierungen und Restriktionen veranlasst, die einem nicht passen, fahndet man nach Uneindeutigkeiten, Unvollkommenheiten und Widersprüchen im Expertenwissen und wertet diese als Systemversagen. Gleichzeitig hofft man auf die wissenschaftliche Absolution politischer Abwarte- und Abstinenzstrategien durch willfährige Gegen- oder Pseudoexperten. Auch die Wissenspolitik der Leugnerbewegung ist also ganz offensichtlich von der Vorstellung geleitet, dass die Politik der Autorität des Expertenkonsenses unbedingt folgen werden muss ...

\section{Getrennte Sphären}

Dennoch: Ohne dies im Mindesten zu wollen, verweist die faktenscheue Fundamentalopposition auf ein grundlegendes Problem, nämlich, dass die Macht des (wissenschaftlichen) Wissens eine Politik der Alternativlosigkeit unterstützen kann. Und selbst dann, wenn die Politik auf diese Option verzichtet, erhöht sich in jedem Fall der Druck auf alle Beteiligten, eigene Werte und Interessen als Ausdruck objektiven, besseren Wissens darzustellen. Auf solche Weise steigt die Chance, dass politische Konflikte als wissenschaftsähnlicher Disput ausgetragen werden.

Mit Blick auf die Folgeprobleme dieser Versachlichung wäre wohl eine (Re-)Politisierung politischer Konflikte zu fordern. Das bedeutet natürlich nicht, dass man in politischen Streitfragen auf wissenschaftliche Expertise grundsätzlich verzichten sollte - das wäre Irrsinn. Es geht jedoch um die Einsicht, dass Politik und Wissenschaft voneinander getrennte Sphären sind: So wenig sich wissenschaftliche Wahrheit nach der Mehrheitsmeinung richtet, so wenig ist es Aufgabe der Politik, die Wahrheit zu vollstrecken. In der Politik geht es in erster Linie darum, ein breites Spektrum an Meinungen und Betroffenheiten zu berücksichtigen. Auf diese Weise kommt man in der Politik zu Positionen, die nicht beanspruchen können, wahr zu sein, die jedoch ein gewisses Maß an Gemeinsinn repräsentieren und dadurch mit Zustimmung rechnen können. 